\title{
Que révèlent les médias sociaux au sujet de la crise des opioïdes au Canada?
}

\author{
Semra Tibebu, B. Sc. (1,2); Vicky C. Chang, M. Sc. (1,2); Charles-Antoine Drouin, B.A. (3); Wendy Thompson, M. Sc. (2); \\ Minh T. Do, Ph. D. $(1,2,4)$
}

\section{Diffuser cet article sur Twitter}

\section{Résumé}

Nous avons utilisé les médias sociaux comme source de données potentielle pour obtenir de l'information en temps réel sur l'usage des opioïdes et sur les perceptions entourant ces substances au Canada. Nous avons recueilli des messages sur Twitter au moyen d'une plateforme d'analyse des médias sociaux entre le 15 juin et le 13 juillet 2017, puis nous les avons analysés afin d'y déceler les thèmes récurrents. Nous avons souvent relevé des messages concernant l'usage d'opioïdes à des fins médicales ainsi que des commentaires sur les efforts d'intervention déployés par le gouvernement du Canada dans le cadre de la crise des opioïdes. Les résultats de l'étude pourraient aider à orienter les pratiques en santé publique ainsi qu'à soutenir les intervenants communautaires dans leurs efforts pour contrer la crise.

Mots-clés : opioüdes, Twitter, usage et perceptions, Canada

\section{Introduction}

En Amérique du Nord, le nombre de décès, d'hospitalisations et de surdoses liés aux opioïdes a augmenté au cours des dernières années $^{1,2}$. Au Canada, le taux d'hospitalisation à la suite d'une intoxication aux opioides pour l'ensemble des groupes d'âge a augmenté de plus de $30 \%$ entre 2007 et 2016, s'établissant à un peu moins de 16 hospitalisations par 100000 personnes $^{3}$. En 2016, on a comptabilisé 2861 décès liés aux opioïdes au Canada ${ }^{4}$. Toutes les provinces ont continué à enregistrer en 2017 des hausses marquées du nombre de décès liés aux opioïdes ${ }^{5-7}$. Disposer de données obtenues rapidement sur les surdoses d'opioïdes serait précieux pour suivre les tendances et aider à prendre des mesures efficaces pour contrer la crise.

Les méthodes habituelles pour étudier l'usage d'opioïdes à l'échelle du Canada sont les enquêtes nationales et les bases de données administratives sur les décès et les surdoses liés aux opioïdes ${ }^{3,6}$. Bien qu'elles fournissent de bons renseignements, ces sources présentent certaines limites, comme le temps nécessaire pour accéder aux données ou publier des résultats, un biais dans les réponses influençant les résultats d'enquête et le manque de détails sur le contexte entourant l'usage des opioïdes.

Les médias sociaux ont déjà été utilisés pour obtenir des données sur certaines questions urgentes en santé publique $e^{9-12}$. Des études antérieures ont utilisé les médias sociaux pour faire le suivi épidémiologique de maladies et sonder les réactions du public sur certains efforts de promotion de la santét-8. Au cours des dernières années, le recours à Twitter en recherche a augmenté (en comparaison avec d'autres médias sociaux) en raison du volume élevé de gazouillis et de la facilité
Points saillants

- Les messages les plus fréquents concernaient l'usage médical personnel d'opioïdes, les plus souvent cités étant la morphine, l’oxycodone et la codéine. Peu de messages portaient sur l'usage récréatif ou illégal de ces substances.

- La question des répercussions dans la collectivité, comme le fait de voir des toxicomanes consommer en public ou des seringues retrouvées au sol, ainsi que des histoires personnelles en lien avec des cas de surdose, ont été relevées.

- Dans de nombreux messages, l'auteur exprimait son opinion quant au manque d'action du gouvernement pour régler la crise des opioïdes.

- Twitter peut être utile pour sonder l'opinion publique sur la crise des opioïdes et l'usage de ces substances à des fins médicales.

d'accès aux données et de recherche parmi ces dernières dans Twitter?

Dans le contexte actuel de la crise des opioïdes, les perceptions du public et l'usage rapporté d'opioïdes par les adeptes de Twitter au Canada pourraient façonner les interventions dans la lutte contre la crise et connaître les réactions des utilisateurs de Twitter à l'égard des efforts déployés. Notre étude cible les données de Twitter qui 
touchent l'usage d'opioïdes et les perceptions entourant ceux-ci au Canada.

\section{Méthodologie}

L'entreprise Nexalogy de Montréal (Québec) qui fournit des services d'analyse sociale a recueilli des données de Twitter entre le 15 juin et le 13 juillet 2017. Cette période a été choisie compte tenu du nombre croissant de décès liés aux opioïdes ayant été enregistrés au Canada dans les mois précédents $^{5-7}$. Pour concevoir la stratégie de recherche de l'étude (réalisée en anglais), des termes génériques courants, des noms de marque et des mots du langage familier associés aux opioïdes ont été sélectionnés à partir de la littérature ainsi que dans Google (https://www.google.ca/) et dans le Urban Dictionary (https://www.urbandictionary.com//13,14. Nous avons utilisé Nexalogy pour effectuer des recherches préliminaires dans Twitter à l'aide des termes désignant les opioïdes et les termes menant à au moins cinq gazouillis sur l'usage d'opioïdes ou les perceptions entourant ceux-ci ont été retenus pour la stratégie de recherche finale (encadré 1). Les gazouillis retenus étaient géolocalisés au Canada ou avaient été diffusés par des

\section{ENCADRÉ 1}

Termes de recherche ayant servi à la collecte de données dans Twitter

fentanyl
oxycontin
opioid
oxycodone
oxy
vicodin
hydromorphone
hydrocodone
morphine
methadone
percocet
codeine
heroin
"Tylenol 3"
vikes
percs
“codeine cough syrup”
“drinking lean”

utilisateurs qui, selon leur profil, résidaient au Canada. Étant donnée la difficulté à établir avec certitude le contexte dans lequel un gazouillis est partagé, tous les gazouillis partagés ont été exclus.

Après avoir téléchargé les messages, nous avons exclu : les messages non pertinents portant sur des nouvelles diffusées par des sociétés journalistiques ou des organismes de santé, les messages contenant des termes associés aux opioïdes dans le nom d'utilisateur seulement (pas dans le contenu), les messages en double et ceux contenant des nombres et des caractères plutôt que du texte. Lorsque la source ou l'intention du message n'était pas claire, nous avons examiné les liens vers les gazouillis initiaux (accompagnant les messages Twitter) pour déterminer la pertinence du gazouillis.

Un schéma global de codage a été établi à partir de la littérature et de termes, phrases et thèmes récurrents dans les messages. Les deux principaux thèmes, " usage » et " perceptions ", étaient mutuellement exclusifs, selon un schéma déjà utilisé dans une étude qualitative sur la mention de termes d'opioïdes dans Twitter ${ }^{14}$. Les messages Twitter ont été lus à maintes reprises, ce qui a permis de diviser les deux principaux thèmes du schéma de codage en sous-catégories, puis de modifier ces dernières jusqu'à ce que tous les messages entrent dans une seule catégorie, les souscatégories étant elles aussi mutuellement exclusives $^{15}$. Tous les messages ont été codés en conséquence. Deux chercheurs ont examiné l'ensemble des messages et ont discuté de toute divergence relevée afin d'établir un consensus, demandant au besoin l'aide d'un troisième chercheur. Nous avons calculé la fréquence de chaque thème.

\section{Résultats}

Au total, 2602 gazouillis correspondant à la stratégie de recherche ont été retenus. De ce nombre, 1776 ont été exclus après avoir été jugés non pertinents à la suite d'un examen manuel. L'ensemble final de données comptait 826 messages : 148 portant sur l'usage d'opioïdes et 678 sur les perceptions entourant ces substances.

\section{Messages sur l'usage d'opioïdes}

De tous les messages en lien avec l'usage d'opioïdes, 37 (25\%) faisaient mention de la morphine, 29 (20\%) de l'oxycodone, 30 (20\%) de la codéine et 33 (22\%) de produits contenant des opioïdes et de l'acétaminophène. Dix messages portaient sur les surdoses, dont huit comportaient des commentaires sur la consommation par une autre personne.

La question de l'usage d'opioïdes à des fins médicales a souvent été relevée ( $\mathrm{n}=70$; $67 \%$ ) dans les messages, laissant plus fréquemment entendre des sentiments négatifs ( $\mathrm{n}=15 ; 42 \%$ ) que positifs ( $\mathrm{n}=13$; $36 \%$ ). La morphine a été mentionnée dans 27 (39 \%) messages sur l'usage à des fins médicales (tableau 1).

Les messages commentant l'usage d'opioïdes par d'autres personnes portaient, sensiblement dans une même mesure, sur l'incidence de cette consommation sur des amis et des proches ( $\mathrm{n}=23 ; 52 \%$ ) et sur la constatation de l'usage de drogues dans des espaces publics ( $\mathrm{n}=21 ; 48 \%$ ). Par exemple, deux messages indiquaient la découverte de seringues dans le quartier (tableau 1).

\section{Messages sur les perceptions entourant les opioïdes}

Dans les messages sur les perceptions entourant les opioïdes, le terme " héroïne " était utilisé le plus souvent ( $\mathrm{n}=203$; $30 \%)$, suivi de "fentanyl » $(\mathrm{n}=184$; $27 \%)$ puis d'« opioïdes » $(n=150 ; 22 \%)$.

Des commentaires sur la crise des opioïdes ont été formulés dans 318 (47\%) messages sur les perceptions. De ces messages, 173 (54\%) contenaient des opinions et des faits au sujet de la crise tandis que 129 (41\%) exposaient des sentiments liés à la crise (tableau 2).

La majorité des sentiments étaient dirigés vers les représentants et leurs efforts pour lutter contre la crise, avec 20 (15\%) messages vers le gouvernement ou les services de police canadiens, 35 (27 \%) messages vers le gouvernement ou les services de police américains et 18 (14 \%) messages exprimant de la colère ou blâmant les entreprises pharmaceutiques et les médecins (tableau 2).

La réduction des méfaits était le sujet de 48 (15\%) messages, dont 22 (46\%) portaient sur la légalisation de l'héroïne sur ordonnance dans le cadre des programmes de traitement contre la dépendance aux opioïdes 
TABLEAU 1

Messages sur l'usage d'opioïdes $(\mathrm{N}=148)$ parmi les utilisateurs canadiens

de Twitter, juin-juillet 2017

\begin{tabular}{|c|c|c|}
\hline Thème & $\begin{array}{c}\text { Fréquence } \\
\text { n (\%) }\end{array}$ & Exemple de message \\
\hline $\begin{array}{l}\text { Usage } \\
\text { personnel }\end{array}$ & $104(70)$ & \\
\hline Médical & $70(67)$ & $\begin{array}{l}\text { Had a migraine from hell, now l'm a limp noodle on the couch, thanks percocet! } \\
\text { [Migraine de l'enfer, je suis comme une nouille sur le sofa, merci percocet!] } \\
\text { I think that codeine is finally kicking in, headache is retreating } \\
\text { [Je pense que la codéine fait enfin effet, mon mal de tête s'en va] } \\
\text { [twitter handle] I'm on codeine for it but it's making me so sick that l'm just taking the pain like a man } \\
\text { [[pseudonyme] Je prends de la codéine pour ça, mais ça me rend tellement malade que je subis la douleur comme un homme] }\end{array}$ \\
\hline Récréatif & $10(10)$ & $\begin{array}{l}\text { Fentanyl and chill } \\
\text { [Fentanyl et chill] } \\
\text { Secret [you] haven't told many; I did heroin and wanted to get addicted } \\
\text { [Petit secret entre nous, j'ai pris de l'héroïne et je voulais devenir toxico] } \\
\text { Gassing up on lean and good percs } \\
\text { [Je me pompe avec du lean et des bons percs] }\end{array}$ \\
\hline Inconnu & $24(23)$ & $\begin{array}{l}\text { I'm high on life and codeine. Can't forget that } \\
\text { [Je suis drogué de vie et de codéine. Peux pas oublier ça] } \\
\text { [twitter handle] Funny story I overdosed on codeine once and now I can't get a prescription anymore lol (not actually... } \\
\text { [[pseudonyme] Histoire comique j'ai fait une surdose de codéine une fois et maintenant je peux plus avoir de prescription (pas } \\
\text { vraiment...] } \\
\text { Found my codeine pills, time [to] down the bottle } \\
\text { [Trouvé mes pilules de codéine, c'est le temps de caler la bouteille] }\end{array}$ \\
\hline $\begin{array}{l}\text { Usage par } \\
\text { autrui }\end{array}$ & $44(30)$ & \\
\hline $\begin{array}{l}\text { Connais- } \\
\text { sances }\end{array}$ & $23(52)$ & $\begin{array}{l}\text { He said he was aware of risks of \#Fentanyl but figured that odds were it wouldn’t be him, he was wrong and almost died } \\
\text { [Il a dit qu'il connaissait les risques du \#fentanyl mais s'est dit que ça tomberait pas sur lui, il s'est trompé et est presque mort] } \\
\text { I know people who “took as directed” and medicated themselves into an opioid coma } \\
\text { [Je connais des gens qui en ont pris « tel que prescrit » et qui sont tombés dans un coma d'opioïdes] } \\
\text { [twitter handle] I had a friend pass away taking a [fentanyl] pill pressed to look like oxy 80, thank you for telling this story } \\
\text { [[pseudonyme] J'ai un ami qui est mort parce qu'il a pris une pilule [de fentanyl] qui ressemblait à du oxy } 80 \text {, merci d'avoir raconté } \\
\text { cette histoire] }\end{array}$ \\
\hline $\begin{array}{l}\text { Dans des } \\
\text { lieux } \\
\text { publics }\end{array}$ & $21(48)$ & $\begin{array}{l}\text { Some kid just walked up to me and asked if I had any percs... } \\
\text { [Un jeune vient juste de m'approcher et de me demander si j'avais des percs...] } \\
\text { You know it's a [bad] day when an obvious heroin addict accidentally gives you a used bloody needle cap when he hands you his } \\
\text { change } \\
\text { [On sait que c'est une mauvaise journée quand une personne de toute évidence héroïnomane vous remet accidentellement un } \\
\text { capuchon de seringue taché de sang avec sa monnaie] } \\
\text { There's heroin needles on the bus \#sudburyprobs \#sudburybuses } \\
\text { [Il y a des seringues d'héroïne dans l'autobus \#problemessudbury \#bussudbury] }\end{array}$ \\
\hline
\end{tabular}


TABLEAU 2

Messages sur les perceptions entourant les opioïdes $(\mathbf{N}=678)$ parmi les utilisateurs canadiens de Twitter, juin-juillet 2017

\begin{tabular}{|c|c|c|}
\hline Thème & $\begin{array}{l}\text { Fréquence } \\
\text { n (\%) }\end{array}$ & Exemple de message \\
\hline Commentaires sur la crise des opioïdes & $318(47)$ & \\
\hline Questions & $16(5)$ & $\begin{array}{l}\text { [twitter handle] they can't just buy fentanyl on the street? } \\
\text { [[pseudonyme] on ne peut pas juste acheter du fentanyl dans la rue?] }\end{array}$ \\
\hline Opinions/faits & $173(54)$ & $\begin{array}{l}\text { Those under } 15 \text { yrs and those over } 65 \text { yrs experience the highest accidental opioid } \\
\text { poisonings. }\end{array}$ \\
\hline
\end{tabular}

[Les moins de 15 ans et les plus de 65 ans sont les plus touchés par les intoxications accidentelles aux opioïdes.]

Statistiques sur les surdoses/décès

$18(10)$

Sentiments

Précautions

Concernant le gouvernement / la police du Canada

Concernant le gouvernement / la police des États-Unis

Blâme/colère à l'endroit des médecins / entreprises pharmaceutiques

Tristesse/peur par rapport à la crise

\section{Autres thèmes}

Messages concernant les États-Unis

Sentiment positif à l'égard des efforts du gouvernement / de la police du Canada

Impact du fentanyl dans la ville de résidence

Importation de fentanyl de Chine

Réduction des méfaits

Discussion sur la légalisation / prescription de l'héroïne

Discussion sur la légalisation du pot
$129(41)$

$17(5)$

22 (46)

13 (27)

Multi overdoses at [correctional centre] in the last 10 days... \#fentanyl \#crisisincorrections

[Nombreuses surdoses au [centre de détention] au cours des 10 derniers jours... \#fentanyl \#criseenprison]

If you party, you can never be on what you get. Watch your friends.

[Si vous faites le party, vous ne pouvez jamais surveiller ce que vous avez eu. Surveillez vos amis.]

[twitter handle] You flooded \#Canada with \#OxyContin..

[[pseudonyme] Vous avez envahi le \#Canada avec l'\#OxyContin...]

Very telling...Shows U.S. world where [US politician's twitter handle] priorities lie...

[Très révélateur...Montre au monde où sont les priorités de [compte de politicien américain]...]

...I understand that the vast majority of opioid usage comes from legal prescription sources

[...Je crois comprendre qu'une bonne partie de la consommation d'opioïdes vient de prescriptions]

This is so scary! \#pei \#drugs \#fentanyl

[Ça fait tellement peur! \#ipe \#drogues \#fentanyl]

[twitter handle] Well all except West Virginia. They're a heroin addict.

[[pseudonyme] Ben, partout sauf en Virgoinie de l’Ouest. Ils sont tous héroïnomanes.]

So thankful for our police officers. Can't begin to imagine what they face every day.

[Tellement reconnaissante envers nos policiers. Je n'imagine même pas ce qu'ils doivent affronter tous les jours.]

Some neighbours say \#fentanyl is a big problem in the area. \#hamont

[Des voisins disent que le \#fentanyl est un gros problème dans le secteur. \#hamont]

[twitter handle] Fentanyl shipped from China as part of the economic genocide plan

[[pseudonyme] Le fentanyl importé de la Chine, partie du programme de génocide économique]

[twitter handle] The fentanyl patch return program is ineffective and hazardous

[[pseudonyme] Le programme de retour de timbre de fentanyl est inefficace et dangereux]

And, to think that Vancouver, B.C. officials are aiming to legislate 'Free Heroin'

[Dire que les aǵents de la Ville de Vancouver veulent légiférer « l’héroïne gratuite »]

[twitter handle] legalized pot will lead to a drop in opioid dependency

[La légalisation du pot à [pseudonyme] fera diminuer la dépendant aux opioïdes]

Suite à la page suivante 
TABLEAU 2 (suite)

Messages sur les perceptions entourant les opioïdes $(\mathbf{N}=678)$ parmi les utilisateurs canadiens de Twitter, juin-juillet 2017

\begin{tabular}{|c|c|c|}
\hline Thème & $\begin{array}{c}\text { Fréquence } \\
\text { n (\%) }\end{array}$ & Exemple de message \\
\hline Commentaires sur les opioïdes en général & $122(18)$ & \\
\hline Positifs & 31 (25) & $\begin{array}{l}\text { Morphine makes the holy known } \\
\text { [La morphine révèle ce qui est saint] }\end{array}$ \\
\hline Négatifs & $30(25)$ & $\begin{array}{l}\text { If you drug someone with fentanyl you should be SHOT } \\
\text { [Si vous droguez quelqu'un au fentanyl, vous devriez vous faire TIRER] }\end{array}$ \\
\hline Opinions, faits, questions & $62(50)$ & $\begin{array}{l}\text { TIL that in Switzerland there is a program that gives a heroin addict heroin with } \\
\text { prescription! } \\
\text { [J'ai appris qu'en Suisse il existe un programme qui donne aux héroïnomanes de } \\
\text { l'héroïne avec une prescription!] }\end{array}$ \\
\hline Questions & $9(15)$ & $\begin{array}{l}\text { [twitter handle] wait is dope pot or heroin, I get confused } \\
\text { [[pseudonyme] attendez, la dope c'est le pot ou l'héroïne, je suis mêlé] }\end{array}$ \\
\hline Recherche & $7(11)$ & $\begin{array}{l}\text { Fascinating study found CBD had no analgesic or antiemetic effects alone } \\
\text { [Une étude fascinante révèle que le CBD n'a pas d'effets analgésiques ou } \\
\text { antiémétiques seul] }\end{array}$ \\
\hline Discussion médicale & $8(13)$ & $\begin{array}{l}\text { [twitter handle] Specific to ACS chest pain. Do you find any significant difference } \\
\text { in response to morphine or fentanyl? } \\
\text { [[pseudonyme] Au sujet des douleurs du SCA. Y a-t-il des différences importantes } \\
\text { entre la réaction à la morphine et la réaction au fentanyl?] }\end{array}$ \\
\hline Opioïdes n'étant pas le sujet principal & $238(35)$ & \\
\hline Conversations d'ordre général/blagues & $159(67)$ & $\begin{array}{l}\text { I fell asleep and they injected heroin into me, haha good joke guys } \\
\text { [Je me suis endormi et ils m'ont injecté de l'héroïne, haha, bonne blague les gars] }\end{array}$ \\
\hline Référence au milieu du divertissement & $59(25)$ & $\begin{array}{l}\text { She's morphine, queen of my vaccine my love, my love } \\
\text { [paroles de chanson] }\end{array}$ \\
\hline Usage par des célébrités & $20(8)$ & $\begin{array}{l}\text { In 1986, Culture Club singer Boy George was charged in London with heroin } \\
\text { possession. } \\
\text { [En 1986, le chanteur de Culture Club, Boy George, a été accusé à Londres de } \\
\text { possession d'héroïne.] }\end{array}$ \\
\hline
\end{tabular}

tandis que $13(27 \%)$ portaient sur la légalisation du cannabis (tableau 2).

Des commentaires sur les opioïdes et les consommateurs d'opioïdes ont été formulés dans 122 (18\%) messages sur les perceptions entourant les opioïdes (tableau 2). La majorité des messages $(62 ; 50 \%)$ comportaient des opinions et des faits, comme des discussions sur la recherche mettant l'accent sur ces substances. Des sentiments positifs à l'égard des opioïdes ou de leur efficacité ont été exprimés dans 31 (25\%) messages tandis que des sentiments négatifs ont été exprimés dans 30 (25\%) messages. Aucun lien n'a été relevé entre le type d'opioïde dont il était fait mention et le sentiment exprimé.

\section{Analyse}

Notre étude démontre que Twitter permet de mettre en contexte l'usage d'opioïdes à des fins médicales et de donner un aperçu des sentiments du public canadien à l'égard des opioïdes.

La mention très fréquente de la morphine, de l'oxycodone et de la codéine dans les gazouillis portant sur l'usage d'opioïdes cadre avec les rapports indiquant que ces substances sont les opioïdes les plus souvent prescrits ${ }^{16-18}$. Étonnamment, l'usage récréatif d'opioïdes n’a pas souvent été mentionné. Bien que Twitter permette aux utilisateurs de demeurer anonymes, d'autres médias sociaux (p. ex. Instagram) sont plus propices à la divulgation de comportements stigmatisés et illégaux, comme la consommation d'alcool et de cannabis chez les mineurs ${ }^{19,20}$.

Les messages faisant mention de l'usage d'opioïdes par d'autres personnes ont permis d'en savoir davantage sur les répercussions de la crise des opioïdes au sein de la collectivité, par exemple sur les signes de consommation d'opioïdes en public et la découverte de seringues au sol. De plus, dans les messages liés aux perceptions, les utilisateurs de Twitter ont fourni des détails, au moyen de statistiques ou d'opinions personnelles, quant à l'incidence de la crise des opioïdes sur leur ville. Il a été particulièrement intéressant de constater le mécontentement exprimé à l'endroit du gouvernement du Canada, des services de police et des entreprises pharmaceutiques. La majorité des auteurs de ces messages considéraient que ces institutions et organismes étaient la source de la crise ou exprimaient leur déception à l'égard des efforts déployés pour combattre la crise. De tels commentaires au sujet des répercussions sur la collectivité et les opinions sur la crise pourraient permettre aux intervenants communautaires et aux administrations municipales d'en apprendre davantage sur la réaction du public à l'égard des efforts 
déployés pour lutter contre la crise des opioïdes.

\section{Forces et limites}

Il s’agit de la première étude axée sur les attitudes et les comportements liés aux opioïdes dans les médias sociaux au Canada. Elle offre des données pertinentes sur l'expérience des Canadiens par rapport à la crise. Un accès complet aux données de Twitter a été possible dans le cadre de l'étude grâce à Nexalogy, ce qui a permis de recueillir toutes les publications pertinentes. L'utilisation de thèmes précis a également permis d'étudier en détail les sentiments et le contexte associés aux messages Twitter.

L'une des limites importantes de l'étude est l'absence de caractéristiques démographiques et géographiques sur les auteurs des publications. Les prochaines études devraient tenter de connaître l'emplacement, l'âge et le sexe ou genre de l'utilisateur. Le fait de mieux comprendre la distribution - par sexe ou genre, âge et emplacement - de l'usage d'opioïdes et des perceptions liées à ceux-ci permettra d'orienter les futures stratégies d'éducation et de prévention de la consommation afin de veiller à ce que les groupes de population affichant des comportements à risque soient bien renseignés sur le sujet. De plus, la courte période de collecte de données a limité le nombre de messages recueillis ainsi que le nombre de nouvelles au sujet de la crise des opioïdes auxquelles les utilisateurs de Twitter pouvaient réagir. Les prochaines études devraient prévoir de plus longues périodes de collecte afin d'étudier les tendances relatives à l'usage et aux perceptions.

L'utilisation de Twitter en tant que source de données présente d'autres limites. Seul un sous-groupe de la population canadienne utilisant Twitter, les données ne proviennent pas d'un échantillon aléatoire, ce qui réduit le caractère généralisable des résultats. De plus, puisque nous n'avons pas été mesure d'obtenir le nombre total de gazouillis publiés pendant la période de collecte des données, nous n'avons pas pu calculer la prévalence des messages sur les opioïdes publiés par les utilisateurs canadiens de Twitter. Enfin, l'analyse par thème a été fastidieuse. Si l'usage des données de Twitter devenait plus large en santé publique, un logiciel d'analyse par thème, comme NVivo, permettrait d'accélérer l'analyse des données et ainsi l'intervention en santé publique ${ }^{21}$.

\section{Conclusion}

Même si une validation plus poussée est nécessaire, notre analyse des données de Twitter semble être, dans l'ensemble, un outil utile pour mesurer rapidement l'opinion publique sur la crise des opioïdes et l'usage médical des opioïdes.

\section{Conflits d'intérêts}

Les auteurs de l'étude n'ont aucun conflit d'intérêts.

\section{Contributions des auteurs et avis}

ST a contribué à la conceptualisation de l'étude, à la collecte, à l'analyse et à l'interprétation des données ainsi qu'à la rédaction du manuscrit; VC a contribué à l'analyse et à l'interprétation des données ainsi qu'à la révision du manuscrit; MD, WT et CD ont contribué à la conceptualisation de l'étude et à la révision du manuscrit.

Le contenu de cet article et les opinions qui y sont exprimées n'engagent que les auteurs et ne sont pas forcément représentatifs de la position du Gouvernement du Canada.

\section{Références}

1. Ciccarone D. Fentanyl in the US heroin supply: a rapidly changing risk environment. Inter $\mathrm{J}$ Drug Policy. 2017;46:107-111. doi: 10.1016/j.drugp0 .2017.06.010.

2. Fischer B, Russell C, Murphy Y, Kurdyak P. Prescription opioids, abuse and public health in Canada: is fentanyl the new centre of the opioid crisis? Pharmacoepidemiol Drug Saf. 2015;24:1334-1336. doi: 10.1002/pds .3901 .

3. Institut canadien d'information sur la santé (ICIS). Préjudices liés aux opioïdes au Canada : Recueil de graphiques, septembre 2017. Ottawa (Ont.) : ICIS; 2017.

4. Gouvernement du Canada. Rapport national : décès apparemment liés à la consommation d'opioïdes (2016). Ottawa (Ont.) : Gouvernement du Canada; 2017.
5. Agence de protection et de promotion de la santé de l'Ontario. Morbidité et mortalité liées aux opioïdes en Ontario [Internet]. Toronto (Ont.) : Santé publique Ontario; 2017 [consulté le 20 août 2017]. En ligne à : http://www .publichealthontario.ca/fr/dataand analytics/pages/opioid.aspx

6. Alberta Health. Opioids and substances of misuse: Alberta report. 2017 Q2. Edmonton $(\mathrm{AB})$ : Government of Alberta; 2017.

7. British Columbia Coroners Service. Illicit drug overdose deaths in BC: January 1, 2007 - October 31, 2017. Burnaby (BC): Ministry of Public Safety; 2017.

8. Agence de la santé publique du Canada (ASPC). Les comportements de santé des jeunes d'âge scolaire : rapport sur les tendances 1990-2010. Ottawa (Ont.) : ASPC; 2014 [consulté le 20 août 2017]. En ligne à : https:// www.canada.ca/fr/sante-publique /services/promotion-sante/enfance -adolescence/programmes-initiatives /sante-scolaire/comportements-sante -jeunes-scolaire/rapport-tendances -1990-2010.html

9. Capurro D, Cole K, Echavarria MI, Joe J, Neogi T, Turner AM. The use of social networking sites for public health practice and research: a systematic review. J Med Internet Res. 2014; 16(3):e79. doi: 10.2196/jmir.2679.

10. Fung IC, Tse ZT, Fu KW. The use of social media in public health surveillance. Western Pac Surveill Response J. 2015;6(2):3-6. doi : 10.5365/wpsar .2015.6.1.019.

11. Charles-Smith LE, Reynolds TL, Cameron MA, et al. Using social media for actionable disease surveillance and outbreak management: a systematic literature review. PLoS One. 2015; 10(10):e0139701. doi: 10.1371/journal .pone.0139701.

12. Sinnenburg L, Buttenheim AM, Padrez K, Mancheno C, Ungar L, Merchant RM. Twitter as a tool for health research: a systematic review. Am J Public Health. 2017;107(1):e1-8. doi : 10.2105/AJPH.2016.303512. 
13. Cavazos PA, Krauss M, Fisher SL, Salyer P, Grucza RA, Bierut LJ. Twitter chatter about marijuana. J Adolesc Health. 2015;56(2):139-45. doi: 10.1016 /j.jadohealth.2014.10.270.

14. Chan B, Lopez A, Sarkar U. The canary in the coal mine tweets: social media reveals public perceptions of non-medical use of opioids. PLoS One. 2015;10(8):e0135072. doi: 10.1371 /journal.pone.0135072.

15. Hamad EO, Savundranayagam MY, Holmes JD, Kinsells EA, Johnson AM. Toward a mixed-methods research approach to content analysis in the digital age: the combined contentanalysis model and its applications to health care twitter feeds. J Med Internet Res. 2016;18(3):e60. doi: 10.2196/jmir.5391

16. Qualité des services de santé Ontario. 9 millions d'ordonnance : Ce que l'on sait de l'utilisation des opiö̈des prescrits par ordonnance en Ontario. Toronto (Ont.) : Imprimeur de la Reine pour l'Ontario; 2017.

17. Dhalla IA, Mamdami MM, Sivilotti ML, Kopp A, Qureshi O, Juurlink DN. Prescribing of opioid analgesics and related mortality before and after the introduction of long-acting oxycodone. CMAJ. 2009;181(12):891-896. doi: 10.1503/cmaj.090784.

18. Gomes T, Mamdani MM, Paterson JM, Dhalla IA, Juurlink DN. Trends in high-dose prescribing in Canada. Can Fam Physician. 2014;60:826-832.

19. Cavazos-Rehg PA, Krauss MJ, Sowles SJ, Bierut LJ. Marijuana-related posts on Instagram. Prev Sci. 2016;17(6):710720. doi: 10.1007/s11121-016-0669-9.

20. Boyle SC, Earle AM, LaBrie JW, Ballou K. Facebook dethroned: revealing the more likely social media destinations for college students' depictions of underage drinking. Addict Behav. 2017;65:63-67. doi: 10.1016/j.addbeh .2016 .10 .004

21. Zimmer M, Proferes NJ. A topology of Twitter research: disciplines, methods, and ethics. Aslib J Inform Manag. 2014;66(3):250-261. doi: 10.1108/AJIM -09-2013-0083. 